\title{
Corrigendum: Coal as an abundant source of graphene quantum dots
}

Ruquan Ye, Changsheng Xiang, Jian Lin, Zhiwei Peng, Kewei Huang, Zheng Yan, Nathan P. Cook, Errol L.G. Samuel, Chih-Chau Hwang, Gedeng Ruan, Gabriel Ceriotti, Abdul-Rahman O. Raji, Angel A. Martí \& James M. Tour

Nature Communications 4:2943 doi: 10.1038/ncomms3943 (2013); published 6 Dec 2013; Updated 23 Apr 2015

In this Article, the bituminous coal graphene quantum dots (b-GQD) are described throughout this paper as having a crystalline hexagonal structure. Following further careful study of the high-resolution transmission electron microscopy data, this claim is too rigorous, and the b-GQDs instead should be referred to as crystalline. This error does not affect the main findings presented in the paper. In addition, the insets in Fig. 2c showing the fast Fourier transform images of the areas highlighted in the figure panel were inadvertently switched. The correct version of Fig. 2 appears below.

a

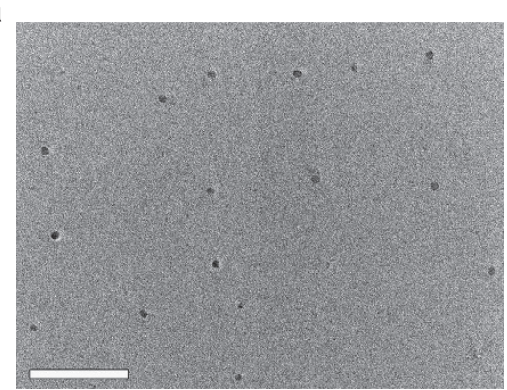

C

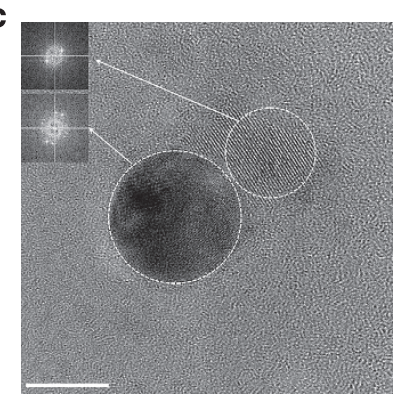

b

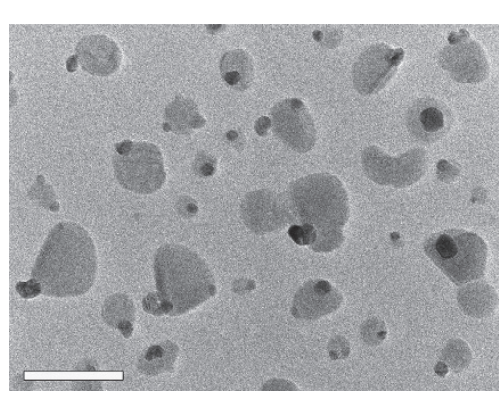

d

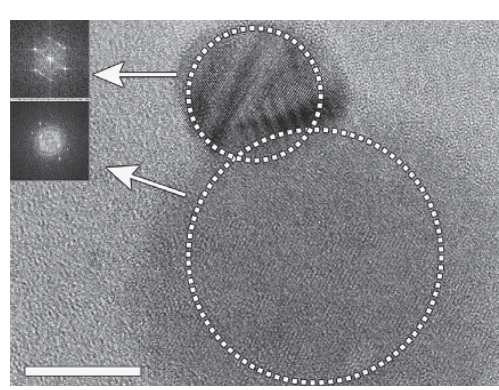

Figure 2 